\title{
Characteristics of Subclinical Coronary Artery Disease in Diabetic Patients without Known Coronary Artery Disease
}

\author{
Kohichiro Iwasaki and Takeshi Matsumoto \\ Department of Cardiology, Okayama Kyokuto Hospital, \\ Kurata, Naka-ku, Okayama, Japan
}

Correspondence should be addressed to: Kohichiro Iwasaki; kiwasaki@kyokuto.or.jp

Received date: 25 November 2013; Accepted date: 23 January 2014; Published date: 14 May 2014

Academic Editor: Alessandro Lupi

Copyright (C) 2014. Kohichiro Iwasaki and Takeshi Matsumoto. Distributed under Creative Commons CC-BY 3.0

\begin{abstract}
Patients with diabetes have a high prevalence of subclinical coronary artery disease (CAD). Our purpose is to study the coronary artery characteristics in diabetic patients. We performed coronary computed tomographic angiography in 374 patients without known CAD. We divided these patients into those with diabetes (diabetes group, $n=138$ ) and those without diabetes (nondiabetes group, $\mathrm{n}=236$ ). We compared coronary calcium score (CCS), prevalence of significant stenosis, coronary plaque, and high-risk plaque (positive remodeling and low attenuation plaque) between the two groups. Patient characteristics were not significantly different between the two groups. The CCS was significantly higher in diabetes group than in nondiabetes group (median with 95\% confidence interval [CI] 202, 95\% CI: $7.5-673$ vs. 60, 95\%CI: $0-291, \mathrm{p}=0.0002)$. The prevalence of significant stenosis $(17.4 \%$ vs. $6.8 \%, \mathrm{p}=0.0014)$ and coronary plaques $(4.3 \pm 4.3$ vs. $3.1 \pm 4.4, p=0.0089)$ were significantly higher in diabetes group compared with nondiabetes group. The prevalence of high-risk plaque $(9.4 \% \mathrm{vs.} 9.7 \%$, $\mathrm{p}=0.92$ ) was not significantly different between the two groups. However, in a multiple logistic regression analysis adjusted for variables associated with a high-risk plaque, only diabetes were significantly associated with a high-risk plaque. Our results showed that diabetic patients had higher CCS, higher prevalence of significant stenosis, and coronary plaque compared with nondiabetic patients. Moreover, diabetes was significantly associated with the presence of highrisk plaque by multivariate analysis
\end{abstract}

Keywords: Diabetes, Coronary artery disease, Coronary computed tomographic angiography High-risk plaque

Cite this Article as: Kohichiro Iwasaki and Takeshi Matsumoto (2014), "Characteristics of Subclinical Coronary Artery Disease in Diabetic Patients without Known Coronary Artery Disease", Journal of Research in Diabetes, Vol. 2014 (2014), Article ID 322292, DOI: 10.5171/2014.322292 


\section{Introduction}

Patients with type 2 diabetes have a two- to four-fold higher risk of a cardiovascular event than nondiabetic patients (Redberg et al., 2002). Previous studies have shown that the risk of myocardial infarction in a diabetic patient is comparable to the risk of recurrent infarction in a nondiabetic patient with a previous myocardial infarction (Haffner et al., 1998). Remarkably, $65 \%$ to $75 \%$ of diabetic patients die of cardiovascular disease (Libby et al., 2005). Thus, there is a clear need to identify diabetic patients who are at risk of cardiovascular events before the onset of symptoms.

Coronary computed tomographic angiography (CCTA) emerged as a promising modality to evaluate coronary artery disease (CAD) noninvasively. Its ability to assess obstructive coronary artery disease has been demonstrated to be excellent compared with conventional coronary angiography (Hamon et al., 2006, Abdulla et al., 2007). Also, its ability to detect coronary plaques and characterize plaque composition has been well appreciated (Schroeder et al., 2001, Achenbach et al., 2004, Leber et al., 2005, Carrascosa et al., 2006).

There are several reports which studied the coronary artery characteristics in diabetic patients (Pundziute et al., 2007, Iwasaki ety al., 2008, Gao etal., 2011, Maffei et al., 2011). However, no study investigated the coronary characteristics associated with vulnerable plaque, so-called high-risk plaque. Thus, our aim is to study the coronary characteristics of diabetic patients, including not only coronary calcium score (CCS), the prevalence of significant stenosis and coronary plaque, but also the prevalence of high-risk plaque, compared with nondiabetic patients.

\section{Methods}

\section{Patients}

From November 2011 through May 2013, we performed CCTA in 521 patients. The following patients were included; 1) patients without known CAD, 2) patients with no symptoms or atypical chest pain. The following patients were excluded; 1) patients who underwent coronary revascularization, 2) patients with acute coronary syndrome, 3) patients with typical chest pain, 4) patients with ECG findings (resting and/or exercise) suggestive of ischemia, 5) patients with serum markers suggestive of myocardial injury, 6) patients with known CAD, and 7) patients with previous hospitalization. Thus, we analyzed CCTA findings of 374 patients.

The patients were divided into two groups according to the presence or absence of type 2 diabetes; diabetes group $(n=138)$ and nondiabetes group $(n=236)$. We assumed that patients had type 2 diabetes when oral diabetic agent or insulin were administered, or when they had a history of type 2 diabetes.

\section{Coronary Computed Tomography Angiography (CCTA)}

We scanned all patients with a 64 multidetector computed tomography (64-MDCT) scanner (SOMATOM Sensation 64 Cardiac, Siemens Medical Solutions and Erlangen, Germany). We administered metoprolol $20 \mathrm{mg}$ before the 64 -MDCT scan in patients with a heart rate $>70$ beats $/ \mathrm{min}$. We also administered sublingual nitroglycerin $0.8 \mathrm{mg}$ before the scan to achieve maximal coronary vasodilation.

We performed a native scan without contrast dye to determine the total calcium burden of the coronary tree (sequential scan with 32X0.6-mm collimation, tube current $60 \mathrm{mAs}$ at $120 \mathrm{kV}$ ). We acquired contrast-enhanced CT angiography data with the use of a spiral scan with $32 \times 0.6-\mathrm{mm}$ collimation, $330-\mathrm{ms}$ gantry rotation, pitch of 0.2 , and tube voltage at $120 \mathrm{kV}$. We acquired a total of 64 overlapping $0.6-\mathrm{mm}$ slices per rotation, with the use of a focal spot periodically moving in the longitudinal direction (z-flying focal spot). We modulated tube current according to the ECG, with a maximum current of 850 to $950 \mathrm{mAs}$ during a time period of 
approximately $330 \mathrm{~ms}$ centered at $375 \mathrm{~ms}$ before the next R-wave, and reduction by $80 \%$ during the remaining cardiac cycle. We injected contrast agent $(60$ to $70 \mathrm{ml} ; 370 \mathrm{mg}$ iodine /ml) intravenously $(4.0 \mathrm{ml} / \mathrm{s})$ followed by a $30-\mathrm{ml}$ saline chaser. We reconstructed transaxial images with the use of an ECG-gated half-scan reconstruction algorithm (temporal resolution $164 \mathrm{~ms}$ ) and kernel B30f.

\section{CCTA Image Interpretation}

We transferred CT data sets to an offline workstation (Aquarius NetStation, Terarecon In, San Meteo, CA, USA) for image analysis. We calculated total calcium score with dedicated software and expressed as Agatston scores, which is a scoring method that calculates the total amount of calcium on the basis of the number, areas, and peak Hounsfield units of the detected calcified lesions (Agatston et al., 1990).

The contrast-enhanced CT angiography data were evaluated by two independent reviewers with maximum intensity, and curved multiplanar reconstruction techniques along multiple longitudinal axes and transversely. We defined calcified plaque as any structure with a density $>130 \mathrm{HU}$ which could be visualized separately from the coronary lumen, assigned to the coronary artery wall, and identified in at least two independent planes. We defined noncalcified plaque as any structure which could be assigned to the coronary artery wall, had a CT density less than the contrast-enhanced coronary lumen but greater than the surrounding connective tissue, and identified in at least two independent planes (Ferencik et al., 2006). We used standard display settings for the evaluation of the contrastenhanced 64-MDCT scans (window width 800 Hounsfield units; window center 250 $\mathrm{HU})$.

We classified plaque calcification as spotty or large. We defined the spotty calcification as $<3 \mathrm{~mm}$ in size on curved multiplanar reformation images, and occupied only one side on cross-sectional images (Ehara et al., 2004). We defined the large calcification as the calcification larger than spotty calcification. We defined the coronary arterial remodeling as a change in the vessel diameter at the plaque site in comparison with the reference segment proximal to the lesion. We defined positive remodeling as the diameter at the plaque site more than $10 \%$ larger than that of the reference segment (Motoyama et al., 2007). In non-calcified plaque or plaque with spotty calcification, we measured the lowest CT number of 5 areas of minimum region of interest using the axial image. We defined low attenuation plaque as CT number less than 30HU (Motoyama et al., 2007). We defined high-risk plaque as a plaque with positive remodeling and low attenuation plaque.

Two observers, who were blinded to the clinical characteristics of studied patients, identified coronary segments by the use of a modified American Heart Association classification (Austen et al., 1975). We classified the segments as normal (smooth parallel or tapering borders), non-significant stenosis (luminal irregularities or $<50 \%$ stenosis), or significant stenosis ( $>50 \%$ stenosis).

\section{Statistical Analysis}

Data are expressed as mean \pm SD. Continuous variables in the laboratory data were compared by two group t-test. Because the data for CCS and prevalence of coronary plaques did not show a normal distribution, the Mann-Whitney test was used to determine the differences between the two groups. Discrete variables were expressed as counts or percentage, and compared with chi-square or the Fisher's exact test. We also performed a multiple logistic regression analysis that included all variables. A p value $<0.05$ was considered to be statistically significant. 


\section{Results}

Clinical characteristics of studied patients are shown in Table 1. Separate analysis in male and female are also performed. Age and the prevalence of hypertension, hyperlipidemia, and chronic kidney disease were not significantly different between the two groups. Laboratory data showed that the value of blood sugar and glycated hemoglobin were significantly higher, and total cholesterol and LDL-cholesterol were significantly lower in diabetes group. The usage of angiotensin converting enzyme inhibitor and angiotensin receptor blocker were significantly prevalent in diabetes group.

Table 1: Patient characteristics

\begin{tabular}{|c|c|c|c|c|c|c|}
\hline & \multicolumn{2}{|c|}{ All } & \multicolumn{2}{|c|}{ Male } & \multicolumn{2}{|c|}{ female } \\
\hline & DM group & $\begin{array}{l}\text { Non-DM } \\
\text { group }\end{array}$ & DM group & $\begin{array}{l}\text { Non-DM } \\
\text { group }\end{array}$ & DM group & $\begin{array}{l}\text { Non-DM } \\
\text { group }\end{array}$ \\
\hline $\mathbf{n}$ & 138 & 236 & 96 & 154 & 42 & 82 \\
\hline Age & $68.9 \pm 8.2$ & $68.8 \pm 10.4$ & $67.8 \pm 8.0$ & $67.1 \pm 10.3$ & $71.1 \pm 7.6$ & $72.1 \pm 9.8$ \\
\hline $\mathbf{p}$ & \multicolumn{2}{|c|}{0.98} & \multicolumn{2}{|c|}{0.58} & \multicolumn{2}{|c|}{0.54} \\
\hline \multicolumn{7}{|l|}{$\begin{array}{l}\text { Risk } \\
\text { Factors }\end{array}$} \\
\hline HT & $92(66.7 \%)$ & $158(66.9 \%)$ & $63(65.6 \%)$ & $98(63.6 \%)$ & $29(69.5 \%)$ & $60(73.2 \%)$ \\
\hline $\mathbf{P}$ & \multicolumn{2}{|c|}{0.96} & \multicolumn{2}{|c|}{0.75} & \multicolumn{2}{|c|}{0.63} \\
\hline HL & $82(59.4 \%)$ & $143(60.6 \%)$ & 57 (59.4\%) & $90(58.4 \%)$ & $25(59.5 \%)$ & $53(64.6 \%)$ \\
\hline $\mathbf{P}$ & \multicolumn{2}{|c|}{0.82} & \multicolumn{2}{|c|}{0.88} & \multicolumn{2}{|c|}{0.58} \\
\hline CKD & $41(29.7 \%)$ & $67(28.4 \%)$ & $25(26.0 \%)$ & $37(24.0 \%)$ & $16(38.1 \%)$ & $30(36.6 \%)$ \\
\hline $\mathbf{P}$ & \multicolumn{2}{|c|}{0.79} & \multicolumn{2}{|c|}{0.76} & \multicolumn{2}{|c|}{0.87} \\
\hline \multicolumn{7}{|c|}{ laboratory data } \\
\hline $\begin{array}{c}\text { BS } \\
\text { (mg/dl } \\
\text { ) }\end{array}$ & $151.5 \pm 60.2$ & $105.9 \pm 30.4$ & $160.8 \pm 52.2$ & $112.0 \pm 17.9$ & $\begin{array}{c}160.8 \pm 52 \\
2\end{array}$ & $112.0 \pm 17.9$ \\
\hline $\mathbf{P}$ & \multicolumn{2}{|c|}{$<0.0001$} & \multicolumn{2}{|c|}{$<0.001$} & \multicolumn{2}{|c|}{$<0.001$} \\
\hline $\begin{array}{c}\text { HbA1c } \\
(\%)\end{array}$ & $6.8 \pm 1.4$ & $5.5 \pm 1.1$ & $6.8 \pm 1.0$ & $5.8 \pm 0.4$ & $7.1 \pm 1.2$ & $5.7 \pm 0.3$ \\
\hline $\mathbf{P}$ & \multicolumn{2}{|c|}{0.0001} & \multicolumn{2}{|c|}{0.005} & \multicolumn{2}{|c|}{0.0012} \\
\hline $\begin{array}{c}\text { TG } \\
\text { (mg/dl }\end{array}$ & $163.7 \pm 108.1$ & $141.6 \pm 85.8$ & $174.7 \pm 121.1$ & $143.2 \pm 94.3$ & $144.3 \pm 66.9$ & $\begin{array}{c}135.5 \pm 61 \\
4\end{array}$ \\
\hline
\end{tabular}




\begin{tabular}{|c|c|c|c|c|c|c|}
\hline ) & & & & & & \\
\hline $\mathbf{P}$ & \multicolumn{2}{|c|}{0.065} & \multicolumn{2}{|c|}{0.072} & \multicolumn{2}{|c|}{0.053} \\
\hline $\begin{array}{c}\text { HDL-C } \\
\text { (mg/dl } \\
\text { ) }\end{array}$ & $55.2 \pm 30.0$ & $57.1 \pm 22.9$ & $52.8 \pm 15.8$ & $54.9 \pm 19.5$ & $63.2 \pm 24.3$ & $\begin{array}{c}60.6 \pm 16 \\
1\end{array}$ \\
\hline $\mathbf{p}$ & \multicolumn{2}{|c|}{0.56} & \multicolumn{2}{|c|}{0.53} & \multicolumn{2}{|c|}{0.46} \\
\hline $\begin{array}{c}\text { LDL-C } \\
\text { (mg/dl } \\
\text { ) }\end{array}$ & $100.3 \pm 33.7$ & $117.5 \pm 37.9$ & $102.6 \pm 30.2$ & $115.1 \pm 36.8$ & $108.0 \pm 27.6$ & $\begin{array}{c}128.0 \pm 27 \\
8\end{array}$ \\
\hline $\mathbf{p}$ & \multicolumn{2}{|c|}{0.0003} & \multicolumn{2}{|c|}{0.022} & \multicolumn{2}{|c|}{0.018} \\
\hline \multicolumn{7}{|c|}{ Medication } \\
\hline aspirin & $54(39.1 \%)$ & $84(35.6 \%)$ & $38(39.6 \%)$ & $50(32.5 \%)$ & $16(38.1 \%)$ & $\begin{array}{c}34 \\
(41.5 \%) \\
\end{array}$ \\
\hline $\mathbf{P}$ & \multicolumn{2}{|c|}{0.49} & \multicolumn{2}{|c|}{0.40} & \multicolumn{2}{|c|}{0.72} \\
\hline statin & $70(50.7 \%)$ & $108(45.8 \%)$ & $49(51.0 \%)$ & 75 (48.7\%) & $21(50.0 \%)$ & $\begin{array}{l}33 \\
(40.2 \%) \\
\end{array}$ \\
\hline $\mathbf{P}$ & \multicolumn{2}{|c|}{0.35} & \multicolumn{2}{|c|}{0.44} & \multicolumn{2}{|c|}{0.21} \\
\hline $\begin{array}{c}\text { ACE- } \\
\text { I/ARB }\end{array}$ & $90(65.2 \%)$ & $120(50.8 \%)$ & $53(55.2 \%)$ & $70(45.5 \%)$ & $37(88.1 \%)$ & $\begin{array}{c}50 \\
(61.0 \%) \\
\end{array}$ \\
\hline p & \multicolumn{2}{|c|}{0.0069} & \multicolumn{2}{|c|}{0.13} & \multicolumn{2}{|c|}{0.0018} \\
\hline $\begin{array}{c}\boldsymbol{\beta} \text {-bloc } \\
\text { ker }\end{array}$ & $18(13.0 \%)$ & $27(11.4 \%)$ & $11(11.5 \%)$ & $18(11.7 \%)$ & $7(16.7 \%)$ & $\begin{array}{c}9 \\
(11.0 \%) \\
\end{array}$ \\
\hline $\mathbf{p}$ & \multicolumn{2}{|c|}{0.65} & \multicolumn{2}{|c|}{0.95} & \multicolumn{2}{|c|}{0.37} \\
\hline $\begin{array}{c}\text { oral } \\
\text { DM } \\
\text { agent } \\
\end{array}$ & $124(89.9 \%)$ & $0(0 \%)$ & $85(88.5 \%)$ & $0(0 \%)$ & $39(92.9 \%)$ & $0(0 \%)$ \\
\hline $\mathbf{p}$ & \multicolumn{2}{|c|}{$<0.0001$} & \multicolumn{2}{|c|}{$<0.001$} & \multicolumn{2}{|c|}{$<0.001$} \\
\hline insulin & $14(10.1 \%)$ & $0(0 \%)$ & $9(9.4 \%)$ & $0(0 \%)$ & $5(11.9 \%)$ & $0(0 \%)$ \\
\hline $\mathbf{p}$ & \multicolumn{2}{|c|}{$<0.0001$} & \multicolumn{2}{|c|}{$<0.001$} & \multicolumn{2}{|c|}{$<0.001$} \\
\hline
\end{tabular}

\section{Abbreviations:}

ACE-I; angiotensin converting ezyme inhibitor, ARB; angiotensin receptor blocker, BS; blood sugar, CCB; calcium channel blocker, CKD; chronic kidney disease, HbA1c; hemoglobin A1c, HDL-C; high-density lipoprotein cholesterol, HL; hyperlipidemia,
HT; hypertension, LDL-C; low-density lipoprotein cholesterol, TC; total cholesterol, TG; triglyceride. 
Figure 1 shows CCS of the two groups. The CCS was significantly higher in diabetes group compared with nondiabetes group (median with 95\% confidence interval (CI) 202, 95\% CI: $7.5-673$ vs. $60,95 \% \mathrm{CI}$ : $0-291, p=0.0002)$. In male, the CCS was significantly higher in diabetes group compared with nondiabetes group $(189,95 \%$ CI: $0-1924$ vs. $54,95 \%$ CI: $0-936, \mathrm{p}<0.001)$. In female, the CCS was significantly higher in diabetes group compared with nondiabetes group (97, $95 \% \mathrm{CI}: 0-1446$ vs. $43,95 \% \mathrm{CI}$ : $0-890, \mathrm{p}<0.001)$.

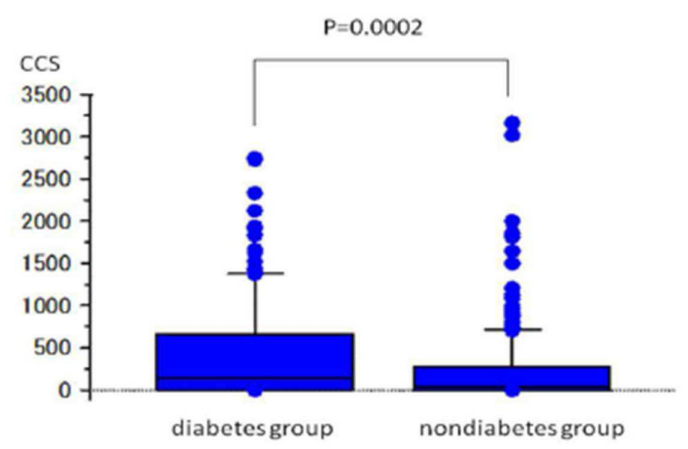

Figure 1: The coronary calcium score of diabetes and nondiabetes group. CCS: coronary calcium score

\section{Figure 1: The coronary calcium score of diabetes and nondiabetes group. CCS: coronary calcium score}

Table 2 shows the prevalence of significant coronary stenosis, coronary plaque, and high-risk plaque. The prevalence of significant coronary stenosis was significantly higher in diabetes group compared with nondiabetes group. The prevalence of patients with any plaque and segments including any plaque was significantly higher in diabetes group compared with nondiabetes group. Segments including calcified plaque were also significantly higher in diabetic group compared with nondiabetes group, but the prevalence of non-calcified plaques was not significantly different between the two groups. The prevalence of positive remodeling and low attenuation plaque were not significantly different between the two groups. Also, the prevalence of high-risk plaque was not significantly different between the two groups. Table 3 shows the results of multiple logistic regression analysis associated with high-risk CCS (CCS $\geq 400$ ), significant stenosis, and high-risk plaque. In a multiple logistic regression, analysis adjusted for variables associated with a high-risk CCS, age and diabetes, were significantly associated with a high-risk CCS. In the same analysis adjusted for variables associated with a significant stenosis, diabetes was the only variable. Moreover, in the same analysis adjusted for variables associated with a high-risk plaque, diabetes was the only variable. 
Table 2: Prevalence of significant coronary stenosis, coronary plaque, and high-risk plaque

\begin{tabular}{|c|c|c|c|c|c|c|}
\hline & \multicolumn{2}{|c|}{ all } & \multicolumn{2}{|c|}{ male } & \multicolumn{2}{|c|}{ female } \\
\hline & DM group & $\begin{array}{l}\text { non-DM } \\
\text { group }\end{array}$ & DM group & $\begin{array}{l}\text { non-DM } \\
\text { group }\end{array}$ & DM group & $\begin{array}{l}\text { non-DM } \\
\text { group }\end{array}$ \\
\hline $\mathrm{n}$ & 138 & 236 & 96 & 154 & 42 & 82 \\
\hline \multicolumn{7}{|l|}{ patients } \\
\hline $\begin{array}{l}\text { with } \\
\text { significant } \\
\text { stenosis }\end{array}$ & $\begin{array}{l}24 \\
(17.4 \%)\end{array}$ & $16(6.8 \%)$ & $14(14.6 \%)$ & $14(9.1 \%)$ & $\begin{array}{l}10 \\
(23.8 \%)\end{array}$ & $2(2.4 \%)$ \\
\hline p & \multicolumn{2}{|c|}{0.0014} & \multicolumn{2}{|c|}{0.18} & \multicolumn{2}{|c|}{$<0.001$} \\
\hline $\begin{array}{l}\text { With any } \\
\text { plaque }\end{array}$ & $\begin{array}{l}116 \\
(84.1 \%) \\
\end{array}$ & $\begin{array}{l}177 \\
(75.0 \%)\end{array}$ & $88(91.7 \%)$ & $126(81.8 \%)$ & $\begin{array}{l}28 \\
(66.7 \%) \\
\end{array}$ & \begin{tabular}{|l|}
51 \\
$(62.2 \%)$ \\
\end{tabular} \\
\hline $\mathrm{p}$ & \multicolumn{2}{|c|}{0.0402} & \multicolumn{2}{|c|}{0.03} & \multicolumn{2}{|c|}{0.62} \\
\hline with PR & $\begin{array}{c}23 \\
(16.7 \%) \\
\end{array}$ & $\begin{array}{c}34 \\
(14.4 \%)\end{array}$ & $19(19.8 \%)$ & 27 (17.5\%) & $4(9.5 \%)$ & $\begin{array}{c}7 \\
(8.5 \%) \\
\end{array}$ \\
\hline $\mathbf{p}$ & \multicolumn{2}{|l|}{0.56} & \multicolumn{2}{|l|}{0.74} & \multicolumn{2}{|l|}{0.74} \\
\hline with LAP & $\begin{array}{l}16 \\
(11.6 \%)\end{array}$ & $\begin{array}{l}30 \\
(12.7 \%)\end{array}$ & $16(16.7 \%)$ & $28(18.2 \%)$ & $0(0 \%)$ & $2(2.4 \%)$ \\
\hline $\mathrm{p}$ & \multicolumn{2}{|l|}{0.75} & \multicolumn{2}{|l|}{0.86} & \multicolumn{2}{|l|}{0.45} \\
\hline $\begin{array}{l}\text { with high- } \\
\text { risk } \\
\text { plaque }\end{array}$ & $13(9.4 \%)$ & $\begin{array}{l}23( \\
9.7 \%)\end{array}$ & $13(13.5 \%)$ & $22(14.3 \%)$ & $0(0 \%)$ & $1(1.2 \%)$ \\
\hline $\mathbf{p}$ & \multicolumn{2}{|c|}{0.92} & \multicolumn{2}{|c|}{0.85} & \multicolumn{2}{|c|}{0.29} \\
\hline \multicolumn{7}{|l|}{ segments } \\
\hline $\begin{array}{l}\text { including } \\
\text { any } \\
\text { plaque }\end{array}$ & $4.3 \pm 4.3$ & $3.1 \pm 4.4$ & $4.2 \pm 3.0$ & $3.2 \pm 2.9$ & $3.2 \pm 3.2$ & $1.9 \pm 2.2$ \\
\hline p & \multicolumn{2}{|l|}{0.0089} & \multicolumn{2}{|l|}{0.011} & \multicolumn{2}{|l|}{0.012} \\
\hline $\begin{array}{l}\text { including } \\
\mathrm{Ca} \\
\text { plaques }\end{array}$ & $3.5 \pm 3.0$ & $2.5 \pm 2.6$ & $3.8 \pm 3.0$ & $2.9 \pm 2.8$ & $3.1 \pm 3.1$ & $1.7 \pm 2.0$ \\
\hline p & \multicolumn{2}{|c|}{0.0007} & \multicolumn{2}{|c|}{0.018} & \multicolumn{2}{|c|}{0.0046} \\
\hline
\end{tabular}




\begin{tabular}{|c|l|l|l|l|l|l|}
\hline $\begin{array}{l}\text { including } \\
\text { non-Ca } \\
\text { plaque }\end{array}$ & $0.4 \pm 0.8$ & $0.3 \pm 0.8$ & $3.1 \pm 3.1$ & $1.7 \pm 2.0$ & $0.1 \pm 0.4$ & $0.2 \pm 0.5$ \\
\hline $\mathbf{p}$ & \multicolumn{2}{|c|}{0.51} & \multicolumn{2}{|c|}{0.43} & & 0.64 \\
\hline
\end{tabular}

\section{Abbreviations:}

Ca; calcified, LAP; low attenuation plaque, PR; positive remodeling

Table 3: The results of multiple logistic regression analysis associated with high-risk coronary calcium score, significant stenosis, and high-risk plaque

\begin{tabular}{|c|c|c|c|}
\hline High-risk coronary & & calcium score & $(\mathrm{CCS} \geq 400)$ \\
\hline Variable & HR & $95 \% \mathrm{CI}$ & $\mathrm{p}$ \\
\hline Age & 1.08 & $1.05-1.12$ & 0.0001 \\
\hline Diabetes & 3.28 & $1.93-5.56$ & 0.0001 \\
\hline \multicolumn{4}{|l|}{ Significant stenosis } \\
\hline Variable & HR & $95 \% \mathrm{CI}$ & $\mathrm{p}$ \\
\hline Diabetes & 3.13 & $1.53-6.37$ & 0.002 \\
\hline \multicolumn{4}{|l|}{ High-risk plaque } \\
\hline Variable & HR & $95 \% \mathrm{CI}$ & $\mathrm{p}$ \\
\hline Diabetes & 2.80 & $1.41-556$ & 0.003 \\
\hline
\end{tabular}

\section{Abbreviations:}

CCS; coronary calcium score, CI; confidence interval, HR; hazard ratio 


\section{Discussion}

Our results showed that diabetic patients had higher CCS, higher prevalence of significant stenosis and coronary plaque compared with nondiabetic patients. A multivariate analysis supported these results. Moreover, in a multiple logistic regression analysis adjusted for variables associated with a high-risk plaque, only diabetes was significantly associated with a high-risk CCS.

In diabetic patients, myocardial ischemia is often asymptomatic and CAD is frequently in an advanced state when it becomes clinically manifest (BARI investigators 1997). Also, previous studies demonstrate that occult CAD is common among asymptomatic diabetic patients, ranging from $20 \%$ in healthier subjects to $>50 \%$ in patients with more complicated diabetes (Carli et al., 2005). Although, the aggressive medical therapy is important, it will remain crucial to identify the individual patient with significant silent coronary artery disease. Thus, tests for early detection of atherosclerotic vascular disease are therefore needed to better assess cardiovascular risk in asymptomatic individuals, the main focus of primary prevention.

There are several studies which investigated the coronary characteristics of diabetic patients compared with nondiabetic patients. Pundziute et al (2007) studied 215 symptomatic patients (86 with diabetes). They found that diabetic patients showed significantly more diseased coronary segments with more non-obstructive plaques. Relatively, more non-calcified and calcified and less mixed plaques were observed in diabetic patients. Iwasaki et al (2008) studied 93 asymptomatic diabetic and 93 age- and sex-matched nondiabetic patients. CCS was significantly higher in diabetic patients. CCS more than 400 was detected in $9.7 \%$ of nondiabetic compared with $36.6 \%$ of diabetic patients $(\mathrm{p}<0.0001)$. Coronary plaques were found in $67.7 \%$ of nondiabetic compared with $91.4 \%$ of diabetic patients $(p<0.0001)$. Significant coronary stenosis was found in $16.1 \%$ of nondiabetic compared with $33.3 \%$ of diabetic patients $(\mathrm{p}=0.0065)$. Gao et al (2011) studied 594 patients with known or suspected CAD (122 with diabetes). CCS was higher in diabetic patients compared with nondiabetic patients. The percentage of patients with CCS more than 400 among diabetic patients was higher than among nondiabetic patients. Diabetic patients had a higher percentage of coronary segments with non-calcified plaque, calcified plaque, and mixed plaque than nondiabetic patients. More diabetic patients had multivessel obstructive disease compared to nondiabetic patients. Maffei et al (2011) studied 1,126 patients without known CAD (147 with diabetes). Diabetic patients showed a higher number of diseased segments, a higher rate of CCS more than 400 , obstructive CAD, and fewer normal coronary arteries as compared to nondiabetic patients. Taken together, previous studies showed that diabetic patients had more atherosclerotic plaque burden manifested by higher CCS, higher prevalence of significant stenosis and higher prevalence of calcified and non-calcified plaques compared with nondiabetic patients. Our results are consistent with these studies.

Recent studies demonstrate that CCTA characteristics of plaques associated with vulnerable plaque are positive remodeling, low attenuation plaque, and spotty calcification (Motoyama et al., 2007, Matsumoto et al., 2007, van Velzen et al., 2011). Other studies show that the patients with positive remodeling and low attenuation plaque are at a higher risk of acute coronary syndrome (Motoyama et al, 2009, Motoyama et al, 2013). Thus, positive remodeling and low attenuation plaque have been regarded as a high-risk plaque. However, there is no study which investigated the prevalence of high-risk plaque in diabetic patients.

Our study showed that the prevalence of high-risk plaque was not significantly different between the two groups. However, 
by multivariate analysis, only diabetes was significantly associated with high-risk plaque. The reason remains to be determined. Fujimto et al (2012) studied the prevalence of high-risk plaque in 1,139 patients with intermediate or high Framingham risk score. For intermediate Framingham risk core group, the prevalence of high-risk plaque was $3.3 \%, 4.9 \%, 9.8 \%$, and $6.5 \%$ in patients with CCS $0,1-250,251$ $500,>500$, respectively. For high Framingham risk core group, the prevalence of high-risk plaque was $7.0 \%, 20.0 \%, 17.1 \%$, and $12.5 \%$ in patients with CCS $0,1-250$, 251-500, >500, respectively. Thus, highest prevalence of high-risk plaque was not observed in patients with CCS $>500$. In our patients, mean CCS was 440.6, and 231.8 in diabetic and nondiabetic patients, respectively. So, Fujimoto's finding may explain the reason why there was no significant difference in the prevalence of high-risk plaque between the two groups. On the other hand, coronary artery calcification signifies the presence of coronary atherosclerosis, and a strong linear correlation exists between total coronary artery atherosclerotic plaque burden and the extent of coronary artery calcification (Rumberger et al., 1995, Sangiorgi et al., 1998). The coronary artery calcification has been found to be the most powerful predictor of cardiac events, providing independent and incremental information over the risk factorbased assessment of the asymptomatic patients (Budoff et al., 2009, Erbel et al., 2010). Because the progression of coronary atherosclerotic change is not homogeneous in the three major coronary arteries, vulnerable plaque may develop even when the CCS is high. Thus, we speculate that large calcium in patients with high CCS might mask the presence of high-risk plaque.

There are some limitations in our study. First, the number of studied patients is relatively small. A study including large number of patients may make the prevalence of high-risk plaque between the two groups significantly different. Second, the ability of 64 MDCT to detect coronary atherosclerotic plaques is not firmly confirmed. One study reported sensitivities of $92.6 \%$ and $96.6 \%$ for accurate characterization of calcified and non-calcified plaques, respectively (Achenbach et al., 2004). They found that the mean CT density between the different plaque types was different, but they appeared to be significant overlap.

In conclusion, our results showed that diabetic patients had higher CCS, higher prevalence of significant stenosis and coronary plaque compared with those without diabetes. Moreover, diabetes was significantly associated with the presence of high-risk plaque by multivariate analysis. Thus, CCTA would be a valuable tool to identify asymptomatic diabetic patients who are at greater risk of cardiovascular events before the onset of symptoms.

\section{References}

1. Abdulla, J., et al. (2007) "64-Multisclice detector computed tomography coronary angiography as potential alternative to conventional coronary angiography: a systematic review and meta-analysis," Eur Heart J, 28 (24) 3042-3050.

2. Achenbach, S., et al. (2004) "Detection of calcified and noncalcified coronary atherosclerotic plaque by contrast-enhanced, submillimeter multidetector spiral computed tomography. A segment-based comparison with intravascular ultrasound," Circulation, 109 (1) 14-17.

3. Agatston, AS., et al. (1990) "Quantification of coronary artery calcium using ultrafast computed tomography," J Am Coll Cardiol, 15 (4) 827-832.

4. Austen, WG., et al. (1975) "A reporting system on patients evaluated for coronary artery disease. Report of the Ad Hoc Committee for Grading of Coronary Artery Disease, Council on Cardiovascular Surgery, American Heart Association," Circulation, 51 (4 Suppl) 5-40. 
5. Budoff, M.J., et al. (2009) "Coronary calcium predicts events better with absolute calcium scores than age-sex-race/ethnicity percentiles: MESA (Multi-Ethnic Study of Atherosclerosis)," J Am Coll Cardiol, 53 (4) 345-352.

6. Carli, Di. and Hachamovitch, R. (2005) "Should we screen for occult coronary artery disease among asymptomatic patients with diabetes?" J Am Coll Cardiol, 45 (1) 50-53.

7. Carrascosa, P.M., et al. (2006) "Characterization of coronary atherosclerotic plaques by multidetector computed tomography," Am J Cardiol, 97 (5) 598-602.

8. Ehara, S., et al. (2004) "Spotty calcification typifies the culprit plaque in patients with acute myocardial infarction: an intravascular ultrasound study," Circulation, 110 (22) 3424-3429.

9. Erbel, R., et al. (2010) "Coronary risk stratification, discrimination, and reclassification improvement based on quantification of subclinical coronary atherosclerosis: the Heinz Nixdorf Recall study," J Am Coll Cardiol, 56 (17) 1397-1406.

10. Ferencik, M., Nieman, K. and Achenbach, S. (2006) "Noncalcified and calcified coronary plaque detection by contrastenhanced multi-detector computed tomography: a study of interobserver agreement," J Am Coll Cardiol, 47 (1) 207 209.

11. Fujimoto, S., et al. (2012) "Coronary computed tomography angiography-based coronary risk stratification in subjects presenting with no or atypical symptoms," Circ J, 776 (10) 2419-2425.

12. Gao, Y., et al. (2011) "Comparison of atherosclerotic plaque by computed tomography angiography in patients with and without diabetes mellitus and with known or suspected coronary artery disease," Am J Cardiol, 108 (6) 809-813.
13. Haffner, S.M., et al. (1998) "Mortality from coronary heart disease in subjects with type diabetes and in nondiabetic subjects with and without prior myocardial infarction," N Engl J Med, 339 (4) 229-234.

14. Hamon, M., et al. (2006) "Diagnostic performance of multislice spiral computed tomography of coronary arteries as compared with conventional invasive coronary angiography: a meta-analysis," J Am Coll Cardiol, 48 (9) 1896-1910.

15. Iwasaki, K., et al. (2008) "Prevalence of subclinical atherosclerosis in asymptomatic diabetic patients by 64-slice computed tomography," Coron Artery Dis, 19 (3) 195201.

16. Leber, A.W., et al. (2005) "Quantitation of obstructive and nonobstructive coronary lesions by 64-slice computed tomography. A comparative study withquantitative coronary angiography and intravascular ultrasound," J Am Coll Cardiol, 46 (1) 147-154.

17. Libby, P., et al. (2005) "National Heart, Lung, and Blood Institute; National Institute of Diabetes and Digestive and Kidney Disease Working Group on Cardiovascular Complications of Type 1 Diabetes Mellitus," Circulation, 111 (25) 3489-3493.

18. Maffei, E., et al. (2011) "Assessment of coronary artery disease and calcified coronary plaque burden by computed tomography in patients with and without diabetes mellitus," Eur Radiol , 21 (5) 944953.

19. Matsumoto, N., et al. (2007) "Prognostic value of non-obstructive CT low-dense coronary artery plaques detected by multislice computed tomography," Circ J, 71 (12) 1898-1903.

20. Motoyama, S., et al. (2007) "Multislice computed tomographic characteristics of coronary lesions in acute coronary syndromes," J Am Coll Cardiol, 50 (4) 319326. 
21. Motoyama, S, et al. (2009) "Computed tomographic angiography characteristics of atherosclerotic plaques subsequently resulting in acute coronary syndrome," J Am Coll Cardiol , 54 (1) 49-57.

22. Motoyama, S., et al. (2013) "Morphologic and functional assessment of coronary artery disease--potential application of computed tomography angiography and myocardial perfusion imaging," Circ J, 77 (2) 411-417.

23. Pundziute, G., et al. (2007) "Noninvasive assessment of plaque characteristics with multislice computed tomography coronary angiography in symptomatic diabetic patients," Diabetes Care , 30 (5) 1113-1119.

24. Redberg, R.F., et al. (2002) "Conference VI:Diabetes and Cardiovascular Disease; Writing Group III: risk assessment in persons with diabetes," Circulation, 105 (18) e144e152.

25. Rumberger, J.A., et al. (1995) “Coronary artery calcium area by electron-beam computed tomography and coronary atherosclerotic plaque area. A histopathologic correlative study," Circulation, 92 (8) 2157-2162.
26. Sangiorgi, G., et al. (1998) "Arterial calcification and not lumen stenosis is highly correlated with atherosclerotic plaque burden in humans: a histologic study of 723 coronary artery segments using nondecdalcifying methodology," J Am Coll Cardiol, 31 (1) 126-133.

27. Schroeder, S., et al. (2001) "Noninvasive detection and evaluation of atherosclerotic coronary plaques with multislice computed tomography." J Am Coll Cardiol, 37 (5) 14301435.

28. The BARI investigators. (1997) "Influence of diabetes on 5-year mortality and morbidity in a randomized trial comparing CABG and PTCA in patients with multivessel disease: the Bypass Angioplasty Revascularization Investigation (BARI)," Circulation, 96 (6) 1761-1769.

29. van Velzen, J.E., et al. (2011) "Comprehensive assessment of spotty calcifications on computed tomography angiography: comparison to plaque characteristics on intravascular ultrasound with radiofrequency backscatter analysis," J Nucl Cardiol, 18 (5) 893-903. 